\title{
Dynamics of an annular Josephson junction in a rotating magnetic field
}

\section{Grønbech-Jensen, Niels; Malomed, Boris A.; Samuelsen, Mogens Rugholm}

\section{Published in:}

Physical Review B

Link to article, DOI:

10.1103/PhysRevB.46.294

Publication date:

1992

Document Version

Publisher's PDF, also known as Version of record

Link back to DTU Orbit

Citation (APA):

Grønbech-Jensen, N., Malomed, B. A., \& Samuelsen, M. R. (1992). Dynamics of an annular Josephson junction in a rotating magnetic field. Physical Review B, 46(1), 294-300. https://doi.org/10.1103/PhysRevB.46.294

\section{General rights}

Copyright and moral rights for the publications made accessible in the public portal are retained by the authors and/or other copyright owners and it is a condition of accessing publications that users recognise and abide by the legal requirements associated with these rights.

- Users may download and print one copy of any publication from the public portal for the purpose of private study or research.

- You may not further distribute the material or use it for any profit-making activity or commercial gain

- You may freely distribute the URL identifying the publication in the public portal 


\title{
Dynamics of an annular Josephson junction in a rotating magnetic field
}

\author{
Niels Grønbech-Jensen \\ Department of Applied Physics, Stanford University, Stanford, California 94305 \\ Boris A. Malomed* and Mogens R. Samuelsen \\ Physics Laboratory I, The Technical University of Denmark, 2800 Lyngby, Denmark \\ (Received 3 January 1992)
}

\begin{abstract}
We study analytically and numerically the dynamics of a solitary fluxon in a long annular damped Josephson junction placed into a rotating magnetic field, which is produced by superposition of two mutually perpendicular ac fields with a phase difference of $\pi / 2$. We demonstrate that the rotating magnetic field drives the fluxon as a traveling wave. The step in the $I-V$ characteristic, corresponding to the velocity of the fluxon coinciding with the phase velocity of the driving wave, is predicted and its size is calculated. It is also demonstrated that the $I-V$ curve has a parabolic profile near the edges of the step. For the case where the fluxon is slowly dragged by a fast traveling wave, the drift velocity is found. The analytical results are in very good agreement with numerical experiments performed on the perturbed sine-Gordon system. Finally the system is analyzed analytically for moderate or small lengths of junctions.
\end{abstract}

\section{INTRODUCTION}

The annular long Josephson junctions (LJJ's), built several years ago, ${ }^{1}$ is a very convenient tool for the experimental study of dynamics of the Josephson solitons (fluxons). Recently, the mechanism of interaction between a LJJ and an external magnetic field lying in the plane of the LJJ has been analyzed theoretically. ${ }^{2-4}$ In particular, the effect of the external magnetic field on the one-fluxon dynamics in an annular LJJ has been considered. It has been demonstrated that the system is governed by the following perturbed sine-Gordon (SG) equation:

$$
\phi_{x x}-\phi_{t t}-\sin \phi=\alpha \phi_{t}-\eta+k B \cos (\Omega t) \sin (k x),
$$

where, as usual, ${ }^{5} \phi, \alpha$, and $\eta$ are the magnetic flux, dissipative constant, and bias current density, respectively. The parameters $B$ and $\Omega$ represent the amplitude and the frequency of the external magnetic field, $x$ and $t$ are the normalized coordinates in space and time, respectively, and $k=2 \pi / L$ is the spatial wave number for a junction with the length $L$. The angle between the direction of the magnetic field and the local magnetic field in the junction is then $k x$, which give rise to the $x$ dependence of the last term on the right-hand side of Eq. (1). The $I-V$ (currentvoltage) characteristic of the model Eq. (1), i.e., the dependence of the average fluxon velocity $u_{0}$ upon the dc bias current density $\eta$, was investigated in Refs. $2-4$. It has been demonstrated that this $I-V$ characteristic contains characteristic constant voltage steps at the values given by the junction length and the external frequency

$$
u_{0}= \pm \Omega / k
$$

(provided $\Omega / k<1$ ), corresponding to the resonance between the circular motion of the fluxon in the annular LJJ with the average angular velocity $u_{0} k$ and the ac field applied to it. Smaller steps at $u_{0}= \pm(n / m) \Omega / k$ have also been found in numerical simulations performed in. ${ }^{4}$ These steps can be interpreted as a manifestation of subharmonic resonance between the circular motion of the fluxon and the ac field. ${ }^{7}$

The objective of the present work is to develop analytical and numerical investigation of the model of an annular LJJ interacting with the rotating magnetic field, which can be realized as the superposition of two spatially orthogonal oscillating magnetic fields with common amplitude and frequency and with the relative phase difference of $\pi / 2$ :

$$
\mathbf{B}=B\left[\mathbf{e}_{1} \cos (\Omega t)+\mathbf{e}_{2} \sin (\Omega t)\right],
$$

where $e_{1} \cdot e_{2}=0$. It is obvious that the rotating magnetic field Eq. (3) gives rise to the following dynamical equation:

$$
\phi_{x x}-\phi_{t t}-\sin \phi=\alpha \phi_{t}-\eta+k B \cos (\Omega t-k x),
$$

i.e., a damped dc-driven SG equation with the additional ac traveling-wave drive, unlike the ac standing-wave drive in Eq. (1).

In Sec. II of this work, we develop an analysis of the motion of a fluxon in the model of Eq. (4) based on the adiabatic perturbation technique. ${ }^{6,8,9}$ We demonstrate that the dependence $u_{0}(\eta)$ in the model also gives rise to a step at the value given in Eq. (2). In contrast to the model Eq. (1), in this work, the step corresponds to the motion of a fluxon with constant velocity Eq. (2), and not just an average velocity. We also analyze the form of the $I-V$ characteristic near the edges of the step, and demonstrate that the segments of the $I-V$ curve abutting to the edges of the constant-voltage step have a parabolic shape (see Fig. 1).

The $I-V$ characteristic intersects the axis $\eta=0$ at a nonzero value $V=V_{\mathrm{dr}}$, i.e., in absence of a dc current, the traveling wave drags the fluxon at some drift velocity $u_{\mathrm{dr}}$. 
If $B$ exceeds a certain threshold value $B_{\mathrm{thr}} \sim \alpha$, the fluxon motion may lock its phase to the driving wave and $u_{\mathrm{dr}}=u_{0}$. If $B$ is smaller than $B_{\mathrm{thr}}$, the fluxon is "drifting" at a velocity: $u_{\mathrm{dr}}<u_{0}$. In Sec. II we at first find $u_{\mathrm{dr}}$ for the case $0<B_{\mathrm{thr}}-B<B_{\mathrm{thr}}$. Then we calculate the drift velocity in the case when it is much smaller than the phase velocity $\Omega$ of the driving wave.

In Sec. III, we display the results of direct numerical simulations of Eq. (4). First of all, we find the $I-V$ characteristic of the system. The numerically calculated characteristic contains a well-pronounced constantvoltage step, exactly corresponding to the one predicted analytically. Also the results of the numerical calculations are in good agreement with the predicted drift velocity of the fluxon.

In Sec. IV we consider the short junction (smaller than or comparable to the Josephson penetration length$L \lesssim 1$ in the present notation). In this case, we use the well-known cnoidal wave solution (a periodic array of strongly overlapped unipolar fluxons) instead of the onefluxon solution. The important circumstance is that the driving wave and the cnoidal wave have the same period, equal to the length of the annular junction. Therefore, they are automatically commensurate. For the short junctions we also predict the existence of constantvoltage steps and calculate their sizes. In the general case, this can only be done implicitly, but for very short junctions, we obtain explicit expressions.

\section{DRIVING THE FLUXON BY A TRAVELING WAVE}

\section{A. The stationary motion}

Regarding the driven kink as a solution to the unperturbed sine-Gordon system [left-hand side of Eq. (4)] we have the wave form of the mode as

$$
\phi=4 \tan ^{-1}[\gamma(\dot{\xi})(x-\xi)],
$$

where $\xi$ is the position of the fluxon and $\gamma(\dot{\xi})=\left(1-\dot{\xi}^{2}\right)^{-1 / 2}$ is the inverse Lorentz contraction of the wave profile. Using this solution to describe the fluxon dynamics in the junction, we get the equation of motion for the collective coordinate $\xi$ of the fluxon ${ }^{4,8}$

$$
\frac{d}{d t}[\dot{\xi} \gamma(\dot{\xi})]=-\alpha \dot{\xi} \gamma(\dot{\xi})-\frac{\pi}{4} \eta-\frac{\pi}{4} k B \Gamma \cos (k \xi-\Omega t),
$$

where $\Gamma$ includes information of the dispersion relation of the linear mode driven by a traveling wave $\mathrm{e}^{4,9}$ as well as the effect of the size of the soliton profile relative to the length of the junction:

$$
\Gamma=\frac{1+k^{2}\left(1-\dot{\xi}^{2}\right)}{\left[\left(1+k^{2}-\Omega^{2}\right)^{2}+(\Omega \alpha)^{2}\right]^{1 / 2}} \operatorname{sech}\left[\frac{\pi k}{2 \gamma(\dot{\xi})}\right) .
$$

First of all we can look for the solutions to this equation corresponding to motion of the kink with constant velocity $u_{0}$ equal to the phase velocity of the driving wave Eq.

(2). In this case, we assume $|\Omega|<k$ and write

$$
\xi=u_{0} t+\theta / k,
$$

where $\theta$ describes the internal phase between the fluxon position and the driving wave. Inserting this into Eq. (6) we find

$$
\cos \theta=-\frac{1}{k B \Gamma}\left[\frac{4 \alpha}{\pi} \frac{\Omega}{k} \gamma\left(\frac{\Omega}{k}\right)+\eta\right] .
$$

The solution given by Eqs. (8) and (9) exist, provided that $|\cos \theta| \leq 1$, i.e., that the bias current $\eta$ is in the region

$-\frac{4}{\pi} \alpha u_{0} \gamma\left(u_{0}\right)-k B \Gamma \leq \eta \leq-\frac{4}{\pi} \alpha u_{0} \gamma\left(u_{0}\right)+k B \Gamma$,

where we for simplicity have assumed $B$ to be positive for the definiteness. These inequalities tell us that there is a step on the dependence of the average velocity of the fluxon $\left(u_{0}\right)$ upon the dc current $(\eta)$, i.e., on the $I-V$. characteristic. The step corresponds to the constant voltage $V_{0}=k u_{0}$ and the center of the step is located at the bias point:

$$
\eta=\eta_{0} \equiv-\frac{4}{\pi} \alpha u_{0} \gamma\left(u_{0}\right)
$$

and the total height of the step is easily found to be

$$
\begin{aligned}
\Delta \eta= & 2 k B\left[1+\left\{\left[\frac{\Omega \alpha}{1+k^{2}-\Omega^{2}}\right]^{2}\right\}^{1 / 2}\right]^{-1} \\
& \times \operatorname{sech}\left[\frac{\pi k}{2 \gamma(\Omega / k)}\right] .
\end{aligned}
$$

This predicted height of the phase-locked step in the $I-V$ curve is exactly the double of the height predicted for the standing-wave driven system. ${ }^{2,4}$ However, we note that the assumption Eq. (8) made in order to obtain the result, is exactly fulfilled for the traveling-wave drive, whereas it is an approximation for the standing-wave drive. Therefore, we believe that the expression obtained in this paper is valid for a larger region in parameter space than it was for the system considered in Refs. 2 and 4 . Another thing to note is that the $I-V$ curve is not antisymmetric [around $(V, \eta)=(0,0)$ ] (see Fig. 1) as was the case in the standing-wave driven system. The phase-locked step appears only if the fluxon travels with the same velocity as the driving wave (not the opposite). Thus, fluxons will then give rise to a phase-locked step at the opposite current and voltage values compared to antifluxons.

\section{B. I-V Characteristic near the edges of the phase-locked step}

Let us consider the case when the dc bias $\eta$ is slightly above the upper edge of the step, or slightly below its lower edge [see Eq. (10)]. In these cases we search for the equation of motion for the fluxon in the form

$$
\xi(t)=u_{0} t+\Xi(t),
$$

assuming $\dot{\Xi}^{2}<<u_{0}^{2}$. Inserting this equation into the equation of motion for the collective coordinate [Eq. (6)] and linearizing the resulting expression in $\dot{\Xi}$ and $\ddot{\Xi}$, we arrive at the following effective equation of motion: 


$$
\begin{aligned}
\ddot{\Xi} \gamma^{3}\left(u_{0}\right)= & -\left[\alpha \gamma^{3}\left(u_{0}\right)+\left.\frac{\pi}{4} k B \frac{d \Gamma}{d \dot{\xi}}\right|_{\dot{\xi}=u_{0}} \cos (k \Xi)\right) \dot{\Xi} \\
& -\frac{\pi}{4} \delta \eta-\frac{\pi}{4} k B \Gamma[1+\cos (k \Xi)],
\end{aligned}
$$

where $\delta \eta$ is a small deviation of the $\eta$ value corresponding to the upper or lower edge of the step, and where

$$
\frac{d \Gamma}{d \dot{\xi}}=2 k \dot{\xi} k B \Gamma\left[\frac{\pi}{4} \gamma(\dot{\xi}) \tanh \left[\frac{\pi k}{2 \gamma(\dot{\xi})}\right]-\frac{k}{1+k^{2}-\Omega^{2}}\right] .
$$

Let us assume that the inertia term in Eq. (14) may be neglected if compared to the dissipative term. Then Eq. (14) reduces to the first-order overdamped equation

$$
\left.\dot{\Xi}=-\frac{\pi}{4 \alpha} \gamma^{-3}\left(u_{0}\right)(\delta \eta \mp k B \Gamma) \mid 1-\frac{k B \Gamma}{\delta \eta \mp k B \Gamma} \cos (k \Xi)\right) /\left(1+\left.\frac{\pi}{4} \frac{k B}{\alpha} \gamma^{-3}\left(u_{0}\right) \frac{d \Gamma}{d \dot{\xi}}\right|_{\dot{\xi}=u_{0}} \cos (k \Xi)\right),
$$

where the $\mp$ applies for the cases $\delta \eta>0$ and $\delta \eta<0$, respectively. Using this equation, we may obtain the following rough estimates:

$$
\begin{aligned}
& \doteq \sim \alpha^{-1}\left(1-u_{0}^{2}\right)^{3 / 2} k B \Gamma, \\
& \ddot{\Xi} \sim \alpha^{-2}\left(1-u_{0}^{2}\right)^{3}(k B \Gamma)^{2} L^{-1} .
\end{aligned}
$$

From these estimates, we find that, provided $L \gg k B \Gamma \alpha^{-2}\left(1-u_{0}^{2}\right)^{3 / 2}$, the overdamped assumption holds. In the following treatment this assumption is made.

To determine the form of the $I-V$ characteristic near the edges of the step, we need to find the mean value of $\dot{\Xi}$, i.e., the deviation of the mean velocity of the fluxon from the value $u_{0}$. From the standpoint of Eq. (16) we consider the motion of the overdamped particle in the harmonic potential with the period $L$ in the presence of the additional driving force. Thus, the mean value of $\dot{\Xi}$ is

$$
\langle\dot{\Xi}\rangle= \pm L / T \text {, }
$$

where $T$ is the period of this motion, and the sign in Eq. (19) corresponds to $\delta \eta>0$ and to $\delta \eta<0$, respectively. The brackets denote the time average. The period $T$ can be found in a straightforward way:

$$
T=\left|\int_{0}^{L} \frac{d \Xi}{\dot{\Xi}}\right|
$$

where the expression for $\dot{\Xi}$ is taken from Eq. (16). Next, it is easy to notice that, since $\delta \eta$ is assumed to be sufficiently small, the integral Eq. (20) is dominated by the contribution from small values of $\Xi$, where the integral is "almost divergent." The final result is to the lowest order in $\delta \eta$

$$
\langle\dot{\Xi}\rangle=\mp \frac{\pi}{4 \alpha}\left(1-u_{0}^{2}\right)^{3 / 2}\left(1+\left.\frac{\pi}{4} \frac{k B}{\alpha} \gamma^{-3}\left(u_{0}\right) \frac{d \Gamma}{d \dot{\xi}}\right|_{\dot{\xi}=u_{0}}\right)^{-1} \sqrt{2 \delta \eta k B \Gamma} .
$$

Thus, the voltage is $V=-k\left(u_{0}+\langle\dot{\Xi}\rangle\right)$. Note that this expression remains valid as long as $|\dot{\Xi}|<<\left|u_{0}\right|$. According to Eq. (21), the segments of the $I-V$ curve near the edges of the phase-locked step must have the parabolic shape. As we will demonstrate in the next chapter, this prediction is qualitatively in accord with the results of direct numerical simulations.

\section{C. $V-B$ characteristic $(\eta=0)$ near the edge of the locked step}

As follows from Eq. (10), the traveling wave can drive the fluxon Eq. (5) at the velocity $u_{0}=\Omega / k$ in absence of a dc current $(\eta=0)$, provided the amplitude of the external magnetic field exceeds the threshold value given by $(\Omega<k)$

$$
B_{\mathrm{thr}}=\frac{4}{k \pi \Gamma} \alpha \frac{\Omega}{k} \gamma\left(\frac{\Omega}{k}\right) \text {. }
$$

If $|B|$ is smaller than $B_{\mathrm{thr}}$, the fluxon is dragged by the traveling wave at a velocity $u_{\mathrm{dr}}$ smaller than the velocity of the traveling wave $\left(u_{0}\right)$. In this subsection, we consider the case

$$
|B|=B_{\mathrm{thr}}-\delta B, \quad 0<\delta B<<B_{\mathrm{thr}} .
$$

The analysis proves to be formally identical to the analysis above for the constant voltage step, and the final result can be obtained similar to Eq. (21) in the following form:

$$
\left|u_{\mathrm{dr}}\right|=\left|u_{0}\right|-\frac{\pi}{4 \alpha}\left[k B_{\mathrm{thr}} \Gamma \gamma^{-3}\left(u_{0}\right)\right]\left(1+\left.\frac{\pi}{4 \alpha} \gamma^{-3}\left(u_{0}\right) k B_{\mathrm{thr}} \frac{d \Gamma}{d \dot{\xi}}\right|_{\dot{\xi}=u_{0}}\right)^{-1}\left(2 \frac{\delta B}{B_{\mathrm{thr}}}\right)^{1 / 2} .
$$


Thus, in the overdamped limit, the voltage $(V=-k u)$ depends parabolically on a small deviation $\delta B$ in $B$ below the threshold value $B_{\mathrm{thr}}$. We note that in the parameter region where the overdamped assumption does not hold, more complicated dynamics occur. In particular, hysteretic behavior in the small deviation $\delta B$ is to be expected (this is, of course, also true for $\delta \eta$ in the treatment above).

\section{The slowly dragged fluxon}

We will now look for the drift velocity, assuming $\eta=0$ and $u_{\mathrm{dr}} \ll u_{0}$. In this case it is natural to separate the rapidly oscillating part $\zeta(t)$ and the systematic drift in the equation of motion for the fluxon, i.e.,

$$
\xi(t)=u_{\mathrm{dr}} t+\zeta(t) .
$$

We assume that $u_{\mathrm{dr}}$ is constant, and that $\zeta$ gives no contribution to the average velocity of the fluxon: $\langle\dot{\zeta}\rangle=0$. The subsequent analysis can be developed for the general case, when the instantaneous velocity of the oscillations $\dot{\zeta}$ attains "relativistic" values, $\dot{\zeta}^{2}\left(1-\dot{\zeta}^{2}\right)^{-1} \sim 1$, but the corresponding expressions prove to be extremely troublesome. Therefore, we will confine ourselves to the "nonrelativistic" case, which requires us to assume $k B \Gamma<<$. In this case, the underlying equation of motion Eq. (6) with $\eta=0$ takes the form

$$
\ddot{\xi}=-\alpha \dot{\xi}-\frac{\pi}{4} k B \Gamma_{0} \cos (k \xi-\Omega t),
$$

where $\Gamma_{0} \equiv \Gamma(\dot{\xi}=0)$. Separating the fast oscillations from the slow drift gives us the following set of equations:

$$
\begin{aligned}
& \ddot{\zeta}=-\alpha \dot{\zeta}-\frac{\pi}{4} k B \Gamma_{0} \cos \left[\left(\Omega-u_{\mathrm{dr}} k\right) t\right] \\
& u_{\mathrm{dr}}=-\frac{\pi}{4 \alpha} k^{2} B \Gamma_{0}\left\langle\zeta \sin \left[\left(\Omega-k u_{\mathrm{dr}}\right) t\right]\right\rangle,
\end{aligned}
$$

where the brackets mean time average. The solution to these equations are easily found to be

$\xi=\frac{\pi}{4} \frac{k B \Gamma_{0}}{\Omega^{2}+\alpha^{2}}\left[\cos \left[\left(\Omega-k u_{\mathrm{dr}}\right) t\right]-\frac{\alpha}{\Omega} \sin \left[\left(\Omega-k u_{\mathrm{dr}}\right) t\right]\right]$,

$u_{\mathrm{dr}}=\frac{\pi^{3}}{16 L} \frac{\left(k B \Gamma_{0}\right)^{2}}{\Omega^{3}} \frac{1}{1+(\alpha / \Omega)^{2}} \quad(\eta=0)$,

where Eq. (30) is given to the lowest order in $B$. Thus the $I-V$ curve intersects the $\eta=0$ axis at a voltage $V_{\mathrm{dr}}=-k u_{\mathrm{dr}}$.

Making the separation of the fast oscillations and the slow drift we assumed that the instantaneous velocity of the oscillations were much larger than the drift velocity. In order to make the above treatment valid, this condition yields the following condition on the parameters

$$
L \gg \frac{\pi^{2}}{4} \frac{k B \Gamma_{0}}{\Omega^{2}} \frac{1}{\left[1+(\alpha / \Omega)^{2}\right]^{1 / 2}},
$$

which can always be satisfied for sufficiently long junctions.
We note that if the dc bias $\eta$ is to be taken into account, this can be done quite easily in the nonrelativistic limit of fluxon motion. The analysis is identical to the one performed above for $\eta=0$ and the resulting drift velocity of the fluxon turns out to become

$u_{\mathrm{dr}}=\widetilde{u}+\frac{\pi^{3}}{16 L} \frac{\left(k B \Gamma_{0}\right)^{2}}{(\Omega+\widetilde{\omega})^{3}} \frac{1}{1+[\alpha /(\Omega+\widetilde{\omega})]^{2}} \quad(|\eta|<<1)$,

where the fluxon velocity $\widetilde{u}=\widetilde{\omega} / k$ for $B=0$ is given by

$$
\widetilde{\omega}=-k \pi \eta / 4 \alpha, \quad|\widetilde{\omega}| \ll k,
$$

and where we have assumed that $\Omega \gg \widetilde{\omega}$.

\section{NUMERICAL EXPERIMENTS}

In order to verify the perturbation results obtained by the adiabatic approach of the collective coordinate, we have performed numerical experiments on the system described by Eq. (4). The numerical method for integrating the system has been chosen to be a second-order finite difference method in time and a fourth-order finite difference in space. The integration step sizes in time and space have been varied pending on the chosen system parameters. For simplicity, we have in all cases performed our simulations for a dissipation parameter $\alpha=0.1$.

In Fig. 1 we show simulated $I-V$ curves for the parameters, $L=20$, and $\Omega=0.8 k, k B=0.1$ (solid), and $k B=0$ (dashed). Clearly we observe the predicted symmetric phase-locked step at the frequency of the driving traveling wave (note that the driven fluxon traveling in positive direction gives rise to a negative voltage - a step at a positive voltage would occur if an antifluxon were driven or if the driving wave traveled in negative direction). Also, we observe the predicted parabolic shape of the $I$ - $V$ curve near the edges of the step, as predicted in Sec. II B. In the inset of Fig. 1 we have shown the details of the $I-V$ curve for very small values of the dc bias $\eta$. Here we see the voltage drift (solid curve) corresponding to the drift velocity as described in Sec. II D.

In Fig. 2 we have shown the comparison between the analytically predicted step sizes of Eq. (12) (solid curves) and the step sizes resulting from the numerical experiments (error bars) for different values of system lengths and driving frequencies. As is seen, the prediction is perfect within the resolution of the numerical results for all the tested parameters-even for the relatively short junction with the length $L=5$.

Figure 3 shows the comparison between the analytical prediction (solid curves) of the drift voltage for $\eta=0$ given by Eq. (30) and the resulting voltages from the numerical simulations (markers). The figures show the results for three different values of system lengths $(L=10$, 20 , and 30) in the case of a driving frequency $\Omega=0.5 k$, giving $u_{0}=0.5$ for the phase-locked fluxon motion. The quadratic behavior upon the magnetic field amplitude is very clearly observed for the tested set of system parameters. Also, we find that the hysteretic switch from the drifting mode $\left(u<u_{0}\right)$ to the phase-locked mode $\left(u=u_{0}\right)$ occurs for the shorter junction and not for the longer as 


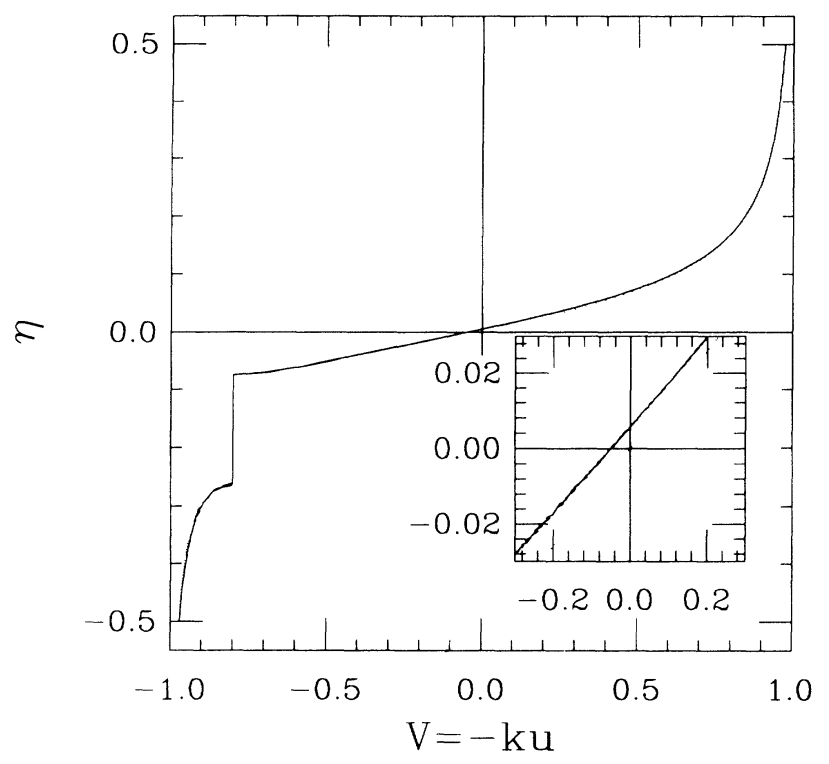

FIG. 1. Normalized $I-V$ curve calculated from the field equation, Eq. (4). Parameters are, $\alpha=0.1, L=20, \Omega=0.8 k$, and $k B=0.1$ (solid line), $k B=0$ (dotted line). The inset shows the details of the $I-V$ curves for very small dc bias current values in the nonrelativistic limit.

noted above in Sec. II. In the case of nonhysteretic behavior, we see that the transition between the two modes obeys the parabolic dependence, predicted in Sec. II C.

In Fig. 4 we have shown the drift voltage as a function of the driving frequency for the case of $L=10, \alpha=0.1$, and $k B=0.01$. Here we find a very close agreement be-

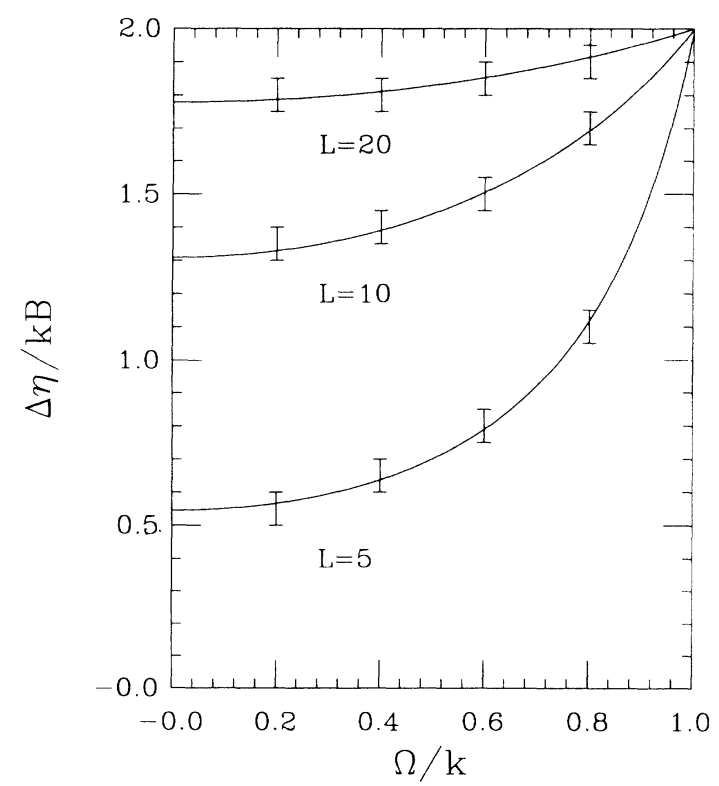

FIG. 2. Comparison between the analytically predicted size of the phase-locked step [Eq. (12)] and the results of numerical simulations on the system defined by Eq. (4) for the system parameters: $\alpha=0.1, k B=0.1$, and $L=5,10,20$, for different values of the driving frequency. Solid lines represent the analytical prediction and the error bars represent the numerical data.
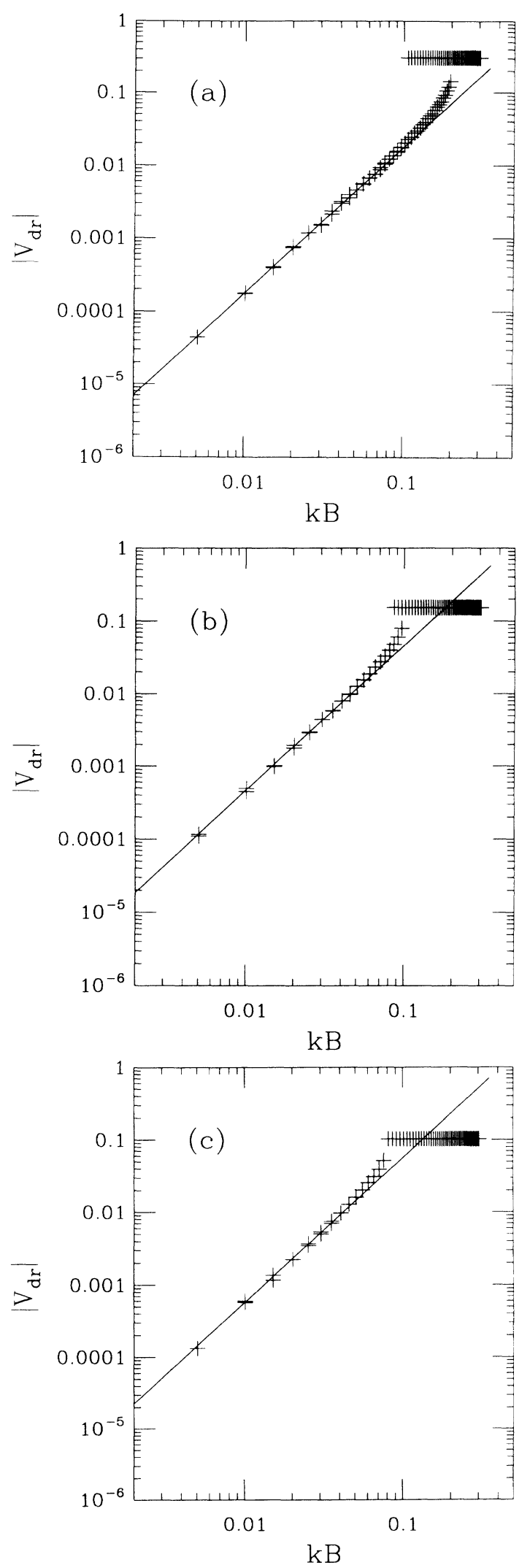

FIG. 3. Comparison between the analytically predicted (solid line) voltage and the numerically measured (markers) average voltage when the fluxon is dragged slowly by the driving wave. The analytical results is given by $V_{\mathrm{dr}}=-k u_{\mathrm{dr}}$, where $u_{\mathrm{dr}}$ is given in Eq. (30). Parameters are $\alpha=0.1, \eta=0, \Omega / k=0.5$, (a) $L=10$, (b) $L=20$, (c) $L=30$. 


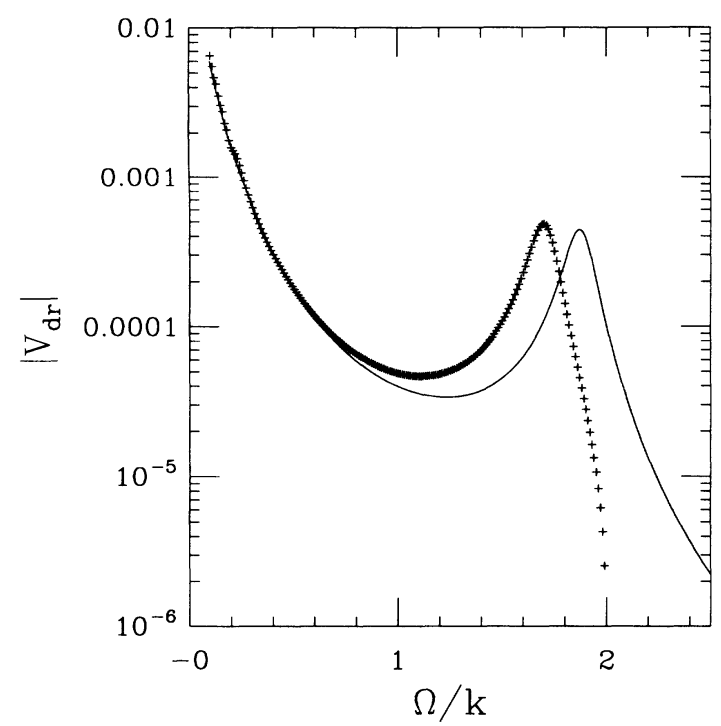

FIG. 4. Comparison between the analytically predicted (solid line) voltage and the numerically measured (markers) average voltage when the fluxon is dragged slowly by the driving wave. The analytical result is given by $V_{\mathrm{dr}}=-k u_{\mathrm{dr}}$, where $u_{\mathrm{dr}}$ is given in Eq. (30). Parameters are, $\alpha=0.1, \eta=0, L=10$, and $k B=0.01$.

tween the analytically predicted voltage (solid line) and the results obtained from the numerical simulations (markers) as long as the frequency is not too close to the resonance of the linear mode given by $\Omega \approx \sqrt{1+k^{2}}$ [see Eq. (7)]. However, the fact that both the analytical curve and the markers show the same overall type of behavior and the fact that they agree very close in the magnitude of the resonance is very satisfactory, since the discrepancy can be easily understood. As is seen from Fig. 4, the main difference between the analytical curve and the numerical measurements is that the resonance of the numerical results is found for a slightly smaller value of the driving frequency than the analytical curve predicts. A discrepancy like this is not very surprising, when the information about the resonance of the linear mode in the perturbation treatment does not include the presence of the soliton, ${ }^{4}$ which is a fairly large perturbation for a junction length of $L=10$. We note, however, that such a discrepancy is particularly clear for relatively short junctions. For the longer junctions, the soliton does not perturb as large a relative part of the junction, and thus, the agreement between the analytical result and the measurements will be much better.

Let us finally note that even in the regions of parameter space, where there are considerable differences between the analytical result and the numerical simulations, we have found that the quadratic dependence of the voltage upon the magnitude of the driving field $(k B)$ is very accurate.

\section{SHORT ANNULAR JUNCTIONS}

\section{A. The general analysis}

In this section, we will analyze the dynamics of the acdriven annular junctions of moderate or short length,
$L \lesssim 1$. Instead of the solitary kink solution of the sineGordon equation, one should in this case base the analysis upon the well-known cnoidal-wave (CW) solution

$$
\phi=\pi-2 \operatorname{am}\left[\gamma(\dot{\xi})(x-\xi) q^{-1}\right],
$$

where am is Jacobi's elliptic amplitude depending on the elliptic modulus $q(0<q<1)$. The solution Eq. (34) must satisfy the periodicity condition

$$
q K(q)=\frac{L}{2 m} \gamma(\dot{\xi})
$$

where $m$ is an arbitrary integer, and $K(q)$ is the complete elliptic integral of the first kind. Note that the change of the magnetic flux $\phi$, when going around the annular junction is $\Delta \phi=2 \pi m$, where $m$ corresponds to the number of trapped fluxons.

To derive the equation of motion for the $\mathrm{CW}$ in the presence of an external magnetic field, a dc current drive, and dissipation, we will make the usual adiabatic assumption, that the CW is sufficiently "rigid," which means that its internal deformation may be neglected, and that it can be governed by the single degree of freedom $(\xi)$. The equation of motion for the $\mathrm{CW}$ collective coordinate is found to be

$$
\begin{aligned}
\frac{d}{d t}\left[E(q) q^{-1} \dot{\xi} \gamma(\dot{\xi})\right]= & -\alpha E(q) q^{-1} \dot{\xi} \gamma(\dot{\xi})-\frac{\pi}{4} \eta \\
& -\frac{\pi}{4} k B \frac{q}{1+q^{2}} \cos (k \xi-\Omega t)
\end{aligned}
$$

Here, the information about the dispersion relation of the driving wave (with spatial wavelength $k$ ), has been neglected, since we expect small amplitude waves to be strongly perturbed by the presence of a fluxon. As in Sec. II, we can immediately predict that a phase-locked step may occur in the $I-V$ characteristic with its center at the bias point $\left(\eta_{0}\right)$ and its size $(\Delta \eta)$ given by

$$
\begin{aligned}
& \Delta \eta=2 k B \frac{q}{1+q^{2}}, \\
& \eta_{0}=-\frac{4 \alpha}{\pi} E(q) q^{-1} \frac{\Omega}{k} \gamma\left(\frac{\Omega}{k}\right),
\end{aligned}
$$

where $q$ is a parameter, given by the implicit expression

$$
q K(q)=\frac{1}{2} L \gamma\left(\frac{\Omega}{k}\right),
$$

$\Omega$ being the frequency at which the fluxon motion is phase locked.

In the nonrelativistic case, the boundary condition Eq. (35) with $m=1$ simplifies to

$$
q K(q)=L / 2
$$

i.e., $q$ is just a parameter dependent on $L$ [Eq. (39) has exactly one solution for any value of $L$ ]. In this case, Eq. (36) is nothing but the one-fluxon equation of motion Eq. (6) with rescaled parameters. However, in the relativistic 
limit, the situation is more complicated because variation of the velocity gives rise to a variation of $q$, and the equation of motion becomes very complicated.

\section{B. The very short junction}

The above analysis strongly simplifies in the limit where $L \ll<1$, when the underlying solution Eq. (34) becomes a nearly linear function. In this case, the boundary condition Eq. (35) with $m=1$ yields

$$
q=\frac{L}{\pi} \gamma(\dot{\xi})
$$

[unless $\pi / L \lesssim(\dot{\xi})$ ], and the equation of motion Eq. (36) takes the form

$$
\ddot{\xi}=-\alpha \dot{\xi}-\frac{L}{4} \eta-\frac{B L}{2} \gamma(\dot{\xi}) \cos (k \xi-\Omega t) .
$$

Solutions to Eq. (42) can be analyzed in a straightforward way, and similarly to the treatments above we find that a phase-locked step occurs, with its center at the bias point $\left(\eta_{0}\right)$ and a total height $(\Delta \eta)$ given by

$$
\begin{aligned}
& \eta_{0}=-\frac{2 \Omega \alpha}{\pi}, \Omega<k \\
& \Delta \eta=4 B \gamma\left[\frac{\Omega}{k}\right], \frac{\pi}{L} \gg \gamma\left(\frac{\Omega}{k}\right) .
\end{aligned}
$$

\section{CONCLUSIONS}

For an annular long Josephson junction coupled to a rotating magnetic field, we have predicted the existence and magnitude of constant voltage steps in the $I-V$ characteristic, when the system is operated in the onefluxon mode. The analysis covers all limiting cases of junction lengths. Further, we have analytically analyzed the shape of the $I-V$ curve near the edges of the phaselocked step as well as for very small values of the dc current drive. Comparisons made between the analytically obtained results and results of direct numerical simulations of the problem have shown very good agreement.

We finally note that long annular Josephson junctions are well within current fabrication capabilities. ${ }^{1,10,11} \mathrm{Ex}$ periments are currently being prepared ${ }^{12}$ in order to verify the phenomena described in this paper.

\section{ACKNOWLEDGMENTS}

One of us (N.G.J.) is grateful to the Otto Mønsteds Fond and the Carlsberg Fondet for financial support during the initial and final part of this work, respectively. Another author (B.A.M.) is indebted to the Technical University of Denmark for hospitality.
*Permanent address: P. P. Shirshov Institute for Oceanology, 23 Krasikov Street, Moscow 117259, U.S.S.R. Address since October 1, 1991: School of Mathematical Sciences, Tel Aviv University, Ramat Aviv 69978, Israel.

${ }^{1}$ A. Davidson, B. Dueholm, B. Kryger, and N. F. Pedersen, Phys. Rev. Lett. 55, 2059 (1985).

${ }^{2}$ N. Grønbech-Jensen, P. S. Lomdahl, and M. R. Samuelsen, Phys. Lett. A 154, 14 (1991).

${ }^{3}$ N. Grønbech-Jensen, P. S. Lomdahl, and M. R. Samuelsen, Phys. Rev. B 43, 12799 (1991).

${ }^{4}$ Niels Grønbech-Jensen, Phys. Rev. B 45, 7315 (1992).

${ }^{5}$ A. Barone and G. Paterno, Physics and Applications of the Josephson Effect (Wiley, New York, 1982).
${ }^{6}$ D. W. McLaughlin and A. C. Scott, Phys. Rev. A 18, 1652 (1978).

${ }^{7}$ Niels Grønbech-Jensen, Boris A. Malomed, and Mogens R. Samuelsen (unpublished).

${ }^{8}$ Yuri S. Kivshar and Boris A. Malomed, Rev. Mod. Phys. 61, 763 (1989)

${ }^{9}$ O. H. Olsen and M. R. Samuelsen, Phys. Rev. B 28, 210 (1983).

${ }^{10}$ A. Davidson and N. F. Pedersen, Appl. Phys. Lett. 44, 465 (1983).

${ }^{11}$ A. Davidson, B. Dueholm, and N. F. Pedersen, J. Appl. Phys. 60, 1447 (1986).

${ }^{12}$ A. Davidson (private communication). 